\title{
Brief Protocol Title
}

National Cancer Institute

\section{Source}

National Cancer Institute. Brief Protocol Title. NCI Thesaurus. Code C132345.

The short, descriptive name for the protocol. 